\title{
Clarifying Demographic Impacts on Embodied and Materially Retained Carbon toward Climate Change Mitigation
}

\author{
Yosuke Shigetomi, ${ }^{*}{ }^{\dagger}$ Hajime Ohno, ${ }^{\ddagger \odot}$ Andrew Chapman, ${ }^{\S}{ }^{\circledR}$ Hidemichi Fujii, ${ }^{\|}$Keisuke Nansai, ${ }^{\perp, \#(0)}$ \\ and Yasuhiro Fukushima ${ }^{\ddagger}$ \\ ${ }^{\dagger}$ Faculty of Environmental Science, Nagasaki University, 1-14 Bunkyo-machi, Nagasaki 852-8521, Japan \\ ${ }^{\ddagger}$ Graduate School of Engineering, Tohoku University, Aramaki Aza Aoba, Aoba-ku, Sendai, Miyagi 980-8579, Japan \\ ${ }^{\S}$ International Institute for Carbon Neutral Energy Research (I2CNER), Kyushu University, 744 Motooka, Nishi-ku, Fukuoka \\ 819-0395, Japan \\ "Faculty of Economics, Kyushu University, 744 Motooka, Nishi-ku, Fukuoka 819-0395, Japan \\ ${ }^{\perp}$ Center for Material Cycles and Waste Management Research, National Institute for Environmental Studies, Japan, 16-2 Onogawa, \\ Ibaraki 305-8506, Japan \\ ${ }^{\#}$ Integrated Sustainability Analysis (ISA), School of Physics, Faculty of Science, The University of Sydney, Camperdown, New South \\ Wales 2006, Australia
}

\section{Supporting Information}

ABSTRACT: Modern lifestyles demand a number of products derived from petroleum-based sources that eventually cause carbon emissions. The quantification of lifestyle and household consumption impacts upon carbon emissions from both the embodied $\mathrm{CO}_{2}$ (EC) and materially retained carbon (MRC) viewpoints is critical to deriving amelioration policies and meeting emission reduction goals. This study, for the first time, details a methodology to estimate both EC and MRC for Japan, focusing on petrochemicals and woody products utilizing the time series input-output table, physical value tables and the national survey of family income and expenditure, leveraging time series input-output-based material flow analysis (IO-MFA), and structural decomposition analysis

(SDA). Findings elucidated hot spots of deleterious consumption by age of householder and the critical factors which underpin them including intensity effects, pattern effects, and demographic shifts over time. Although demographic shifts associated with an aging, shrinking population in Japan decreased EC and MRC, the negative effect reduced in size over time during 19902005. Policy implications identify the potential to mitigate approximately $21 \%$ of required household emission reductions by 2030 through strategies including recycling initiatives and the recovery of carbon from products covered within current recycling laws and hot spot sectors which are not currently considered such as apparel.

\section{INTRODUCTION}

Modern lifestyles depend on petroleum-derived products such as plastics and synthetic rubbers for containers, clothes, and appliances, mainly because of advantages in terms of their lightweight and highly moldable nature. Global plastic production currently accounts for more than 320 million tons $(\mathrm{Mt})$, resulting in global waste issues for the marine environment in addition to human exposure. ${ }^{1-3}$ Another environmental concern is that global greenhouse gas emissions (GHG) related to plastic production will almost quadruple by 2050 compared with 2015 levels unless mitigation strategies including renewable energy, recycling, and demand-management are implemented. ${ }^{4}$ On the other hand, from a carbon metabolism point of view, these petroleum-derived products that are in use or remain fixed in a landfill can be seen as a form of carbon reservoir, alongside forests, wood products, and carbon dioxide $\left(\mathrm{CO}_{2}\right)$ capture and storage (CCS). Therefore, although mechanical and chemical recycling approaches for material recovery could reduce the dependency of production on raw material extraction, most current waste treatment approaches lead to the release of embedded $\mathrm{CO}_{2}$ to the atmosphere during incineration, regardless of the energy recovery process employed. ${ }^{5}$ Reducing $\mathrm{CO}_{2}$ in line with national targets is another crucial issue in order to prevent serious climate change. ${ }^{6-9}$

The carbon physically contained in products at the global and local scale has been analyzed in previous studies. ${ }^{10-14}$

Received: April 30, 2019

Revised: October 30, 2019

Accepted: October 30, 2019

Published: October 30, 2019 
Lauk et al. ${ }^{11}$ found wood products and plastic, respectively, accounted for $61 \%$ and $17 \%$ of total carbon stored in socioeconomic stocks around the globe in 2008, highlighting that per-capita plastic usage has shown high growth since 1900 . Further, Ohno et al. ${ }^{5}$ first addressed the linkage between the amount of carbon contained in products and final consumption. This research elucidated the structure of physical carbon retention for both wooden and petroleum-derived products, namely, materially retained carbon (MRC) induced by Japanese final consumption in 2011, finding that $14.2 \mathrm{Mt}$ of carbon was newly added in Japan, corresponding to $4.1 \%$ of total annual $\mathrm{CO}_{2}$ emissions in the same year if it were all incinerated. Passenger motor cars are the largest contributor, followed by plastic products, cosmetic products, and apparel goods.

Regarding the major contributors to MRC and carbon recoverability, final household consumers may play a critical role for mitigation of the potential $\mathrm{CO}_{2}$ emissions that are released from end-use products by modifying their behavior. ${ }^{5}$ It has been identified that $\mathrm{CO}_{2}$ or GHG emissions generated indirectly from raw material extraction processes through to household consumption, namely, the embodied emissions, contribute significantly toward Japanese lifecycle emissions (i.e., approximately $50 \%$ of the total GHG emissions generated in related supply chains ${ }^{15}$ ), as is also the case in other economically developed nations. ${ }^{16-18}$ In addition, because these nations, notably among them Japan, are experiencing a drastic shift toward an aging, shrinking population, ${ }^{19,20}$ particularly over the last 2 decades, the characteristics of consumption patterns by life-stage varies substantially. These generational consumption pattern differences create diverse environmental burdens due to their supply chains (i.e., environmental footprints). ${ }^{21-24}$ Previous studies suggest that technology and supply chain improvements in energy, manufacturing, and transportation as well as behavioral changes such as becoming car-free, lowering consumption of red meat, and maintaining healthier lifestyles are effective for reducing emissions. ${ }^{25-27}$ In contrast, however, a thorough discussion of the linkages between MRC, household consumption and demographic trends has not been undertaken to date.

Considering further potential carbon reduction in line with the current and future demographic situation mentioned above, this study quantifies MRC and embodied $\mathrm{CO}_{2}$ emissions (EC) in end-use products consumed by age group and simultaneously reveals the impact of aging demographics with respect to societal structures. Figure 1 is a schematic diagram of both MRC and EC as detailed in this study. From a

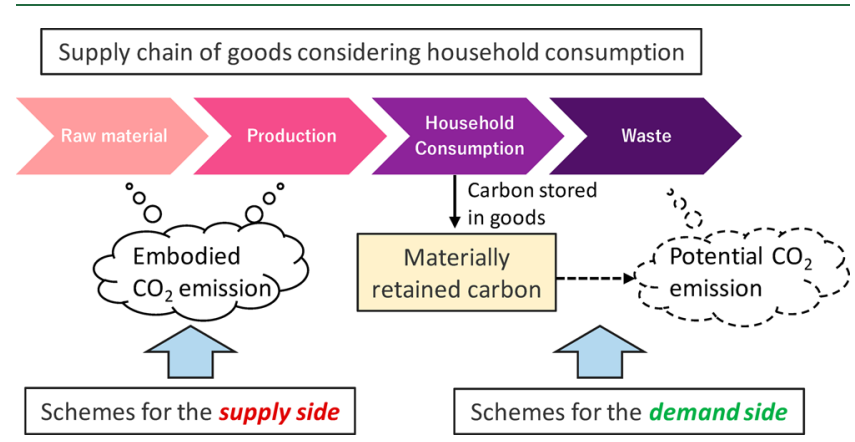

Figure 1. Schematic diagram of embodied $\mathrm{CO}_{2}$ and materially retained carbon in goods consumed by household consumption. consumer point of view, while $\mathrm{EC}$ represents the past $\mathrm{CO}_{2}$ emissions from goods that are currently in use, MRC relates to potential future $\mathrm{CO}_{2}$ emissions from goods, following their consumption. This novel perspective on carbon emissions highlights the importance of adequate policy measures toward climate change mitigation. In addition, to comprehend the dynamics in both EC and MRC, a structural decomposition analysis (SDA) is adopted to clarify the supply and demand factors which drive these changes. SDA is a useful tool for identifying how changes in indicator values impact upon environmental burden changes between two periods. ${ }^{28,29}$ Previous research has adopted this approach to determine changes in energy consumption, ${ }^{30} \mathrm{GHG}^{31-33}$ water consumption, ${ }^{34-36}$ material consumption, ${ }^{37}$ particulate matter $\left(\mathrm{PM}_{2.5}\right){ }^{38}$ and toxic substances. ${ }^{39}$ This study focuses on demographic trends as a deeper demand driver of changes in MRC and EC in line with an aging, shrinking population. To the best of our knowledge, the relationship between these factors and MRC has not yet been clarified. In addition, structural changes in Japanese EC in goods is also yet to be identified, although Japanese direct $\mathrm{CO}_{2}$ emissions from the nationwide, ${ }^{40}$ residential, $^{41}$ manufacturing, ${ }^{42}$ and service sectors ${ }^{43}$ have been investigated. Outcomes obtained through the analysis proposed in this study will therefore contribute toward new carbon abatement opportunities linked with household consumption, lifestyles, and behavior.

\section{METHODOLOGY AND DATA}

2.1. Quantification of Embodied Carbon Dioxide and Materially Retained Carbon Due to Household Consumption. In this study, trends of MRC and EC values were quantified for every 5 years between 1990 and 2005 by multiplying household final consumption with the EC per unit of consumption expenditure (i.e., EC intensity) and MRC per unit consumption of expenditure (i.e., MRC intensity). MRC consists of 9 petroleum- and 2 wood-derived carbons (petroleum-derived; ethylene, propylene, other basic petrochemicals, benzene, toluene, xylene, other aromatic chemicals, methane derivatives recycled thermoplastic resins, and recycled high function resins, wood-derived; timbers and recycled paper) in this study. Note that these do not include bioderived carbons such as sugar cane- and corn-based plastics. The analyzed years are 1990, 1995, 2000, and 2005 cognizant of data limitations detailed in section 4.2. Because the time-series Japan input-output table $(\mathrm{TJIO})^{44}$ compiles monetary inputoutput tables for 1990, 1995, 2000, and 2005 based on 2005 prices of 397 common goods and services (i.e., commodities), utilizing this data is comparable with respect to commodity prices across periods. Next, to identify the relationship between EC, MRC, and demographic trends, this study divides final household demand from the TJIO using the national survey of family income and expenditure (NSFIE), ${ }^{45}$ considering consumption expenditure by household attribute with respect to the age of the highest income earner or person who owns or rents the home (i.e., householder). ${ }^{46}$ In line with previous studies analyzing the impact of demographic shifts on household $\mathrm{CO}_{2}$ emissions, ${ }^{23,47,48}$ consumption expenditure is captured across six age groups of the householder $(\leq 29: 20$ s, $30-39: 30$ s, 40-49:40s, 50-59:50s, 60-69:60s, $\geq 70: 70 s)^{45}$ for each analyzed year. In order to align values and eliminate discrepancies between the IO table (i.e., TJIO), consumer expenditure survey values (i.e., NSFIE) and the difference between producer and consumer-based prices, mathematical 
optimization is undertaken (detailed in a previous study ${ }^{49,50}$ ). Hence, consumption expenditures across the years are presented based on producer prices in 2005 along with the TJIO. The EC and MRC intensities in each of the studied years were estimated based on environmentally extended input-output analysis utilizing the TJIO. ${ }^{51}$ Note that services and capital formation related to commodities consumed by households were ignored in this study because MRC is only associated with goods consumption, this implies that packaging derived from the service sector (e.g., plastic bags from a supermarket) are not included in MRC calculations. Further, packaging which does not reach consumers (i.e., packaging for intermediate-products) was also excluded from MRC. The detailed methodology and data employed for the quantification of EC and MRC is detailed in the Supporting Information.

2.2. Decomposing into Supply and Demand Factors. To comprehend the contribution of various drivers to the changes in both EC and MRC, SDA utilizing the TJIO was adopted. In this study, EC and MRC can be, respectively, decomposed into seven factors as shown in eqs $1-3$.

$$
\begin{aligned}
& \mathrm{EC}=\sum_{h} \hat{\mathbf{q}}_{\mathbf{C O}_{\mathbf{2}}} \mathbf{L y}_{\mathbf{s}}^{h} y_{v}^{h} m^{h} d^{h} n \\
& \mathrm{MRC}^{\text {Petro }}=\sum_{h} \hat{\mathbf{q}}_{\mathbf{M R C}}^{\text {Petro }} \mathbf{L}_{\mathbf{M R C}} \mathbf{y}_{\mathbf{s}}^{h} y_{v}^{h} m^{h} d^{h} n \\
& \mathrm{MRC}^{\text {Wood }}=\sum_{h} \hat{\mathbf{q}}_{\mathbf{M R C}}^{\text {Wood }} \mathbf{L}_{\mathbf{M R C}} \mathbf{y}_{\mathbf{s}}^{h} y_{v}^{h} m^{h} d^{h} n
\end{aligned}
$$

where $\hat{\mathbf{q}}_{\mathbf{C O}_{2}}$, $\hat{\mathbf{q}}_{\text {MRC }}^{\text {Petro }}$, and $\hat{\mathbf{q}}_{\text {MRC }}^{\text {Wood }}$ represent the direct $\mathrm{CO}_{2}$ emissions per unit output, direct input of petroleum-derived carbon per unit output, and that of wood-derived carbon per unit output, respectively. $\mathbf{L}$ represents the Leontief inverse matrix obtained from the TJIO, which indicates the intermediate inputs to produce one unit of output to final demand. $^{52} \quad \mathbf{L}_{\mathbf{M R C}}$ is the modified $\mathbf{L}$ under technological assumptions based on the WIO-MFA, ${ }^{53}$ for estimating the MRC. Superscript $h$ represents household attribute $(h=1: 20 \mathrm{~s}$, $2: 30 \mathrm{~s}, 3: 40 \mathrm{~s}, 4: 50 \mathrm{~s}, 5: 60 \mathrm{~s}, 6: 70 \mathrm{~s})$. $\mathbf{y}_{\mathrm{s}}^{h}$ and $y_{v}^{h}$ represent the household consumption share for commodity and the percapita total consumption volume by attribute, respectively. $m^{h}$ denotes the average number of members in household (family size) by attribute. $d^{h}$ denotes the share of household in the total households by attribute. $n$ is the total number of households. Descriptions of these variables are represented in Table S2 in the Supporting Information. Utilizing these decomposed demographic factors instead of the total population allows for further capturing the effect of demographic changes associated with an aging, shrinking population which leads to an increase in the number of households despite the overall population decline. This is due to societal aging and an increase in single member households, notably in younger and elderly household generations. ${ }^{41}$

To track the contribution of each changing factor toward EC and MRC from year $t$ to year $t+1$, there is no unique solution for how the decomposition should be solved. Here, the total difference in $M R C^{\text {Petro }}$, for instance, is shown in eq 4.

$$
\begin{aligned}
& \Delta \mathrm{MRC}^{\text {Petro }}=\operatorname{MRC}_{(t+1)}^{\text {Petro }}-\operatorname{MRC}_{(t)}^{\text {Petro }} \\
& =\sum_{h} \hat{\mathbf{q}}_{\mathbf{M R C}(t+1)}^{\text {Petro }} \mathbf{L}_{\mathbf{M R C}(t+1)} \mathbf{y}_{\mathbf{s}(t+1)}^{h} y_{v(t+1)}^{h} m_{(t+1)}^{h} d_{(t+1)}^{h} n_{(t+1)} \\
& -\sum_{h} \hat{\mathbf{q}}_{\mathbf{M R C}(t)}^{\text {Petro }} \mathbf{L}_{\mathbf{M R C}(t)} \mathbf{y}_{\mathbf{s}(t)}^{h} y_{v(t)}^{h} m_{(t)}^{h} d_{(t)}^{h} n_{(t)} \\
& =\sum_{h}\left(\Delta \hat{\mathbf{q}}_{\mathbf{M R C}}^{\text {Petro }} \mathbf{L}_{\mathbf{M R C}(t+1)} \mathbf{y}_{\mathbf{s}(t+1)}^{h} y_{v(t+1)}^{h} m_{(t+1)}^{h} d_{(t+1)}^{h} n_{(t+1)}\right. \\
& +\hat{\mathbf{q}}_{\mathbf{M R C}(t)}^{\text {Petro }} \Delta \mathbf{L}_{\mathbf{M R C}} \mathbf{y}_{\mathbf{s}(t+1)} y_{v(t+1)}^{h} m_{(t+1)}^{h} d_{(t+1)}^{h} n_{(t+1)} \\
& +\hat{\mathbf{q}}_{\mathbf{M R C}(t)}^{\text {Petro }} \mathbf{L}_{\mathbf{M R C}(t)} \Delta \mathbf{y}_{\mathbf{s}} y_{v(t+1)}^{h} m_{(t+1)}^{h} d_{(t+1)}^{h} n_{(t+1)} \\
& +\hat{\mathbf{q}}_{\mathbf{M R C}(t)}^{\text {Petro }} \mathbf{L}_{\mathbf{M R C}(t)} \mathbf{y}_{\mathbf{s}(t)} \Delta y_{v}^{h} m_{(t+1)}^{h} d_{(t+1)}^{h} n_{(t+1)} \\
& +\hat{\mathbf{q}}_{\mathbf{M R C}(t)}^{\text {Petro }} \mathbf{L}_{\mathbf{M R C}(t)} \mathbf{y}_{\mathbf{s}(t)} y_{v(t)}^{h} \Delta m^{h} d_{(t+1)^{n}}^{n_{(t+1)}} \\
& +\hat{\mathbf{q}}_{\mathbf{M R C}(t)}^{\text {Petro }} \mathbf{L}_{\mathbf{M R C}(t)} \mathbf{y}_{\mathbf{s}(t)} y_{v(t)}^{h} m_{(t)}^{h} \Delta d^{h} n_{(t+1)} \\
& \left.+\hat{\mathbf{q}}_{\mathbf{M R C}(t)}^{\text {Petro }} \mathbf{L}_{\mathbf{M R C}(t)} \mathbf{y}_{\mathbf{s}(t)} y_{v(t)}^{h} m_{(t)}^{h} d_{(t)}^{h} \Delta n\right)
\end{aligned}
$$

Subscript $t$ denotes the targeted years $(t=1: 1990,2: 1995$, $3: 2000,4: 2005) . \Delta$ is the difference operator. Each additive term in eq 4 represents the contribution to changes in $\mathrm{MRC}^{\text {Petro }}$ associated with changes in each factor, and has $n$ ! decomposition equations. ${ }^{54}$ In this study, each of the contributions were estimated by taking the average of the 7 ! $=5040$ decomposition equations possible. ${ }^{32,54,59}$ The contributions of each change in $\hat{\mathbf{q}}_{\mathrm{CO}_{2}}, \hat{\mathbf{q}}_{\mathbf{M R C}}^{\text {Petro }}, \hat{\mathbf{q}}_{\mathbf{M R C}}^{\text {Wood }}, \mathbf{L}_{\mathbf{M R C}}, \sum_{h} \mathbf{y}_{\mathbf{s}}^{h}$, $\sum_{h} y_{v}^{h}, \sum_{h} m^{h} \sum_{h} d^{h}$, and $n$ denote the intensity effect (EC), intensity effect (petro), intensity effect (wood), supply chain effect, pattern effect, volume effect, size effect, distribution effect, and household effect, respectively. The results of estimating the drivers of $\triangle M R C$ are presented by summing $\Delta \mathrm{MRC}^{\text {Petro }}$ and $\Delta \mathrm{MRC}^{\text {Wood }}$.

Intensity effects and the supply chain effect are suitable indicators for consideration in reducing EC and MRC from the supply side. The former effect measures the change in the sectoral level use of the indicator per unit output while the latter focuses on the changes in the intermediate input structure, enabling the separation of technology change effects. $^{28}$ If the intensity effect (EC) is positive, EC is increased by the growth of direct $\mathrm{CO}_{2}$ emissions from industry in producing commodities from the $\mathrm{TJIO}$. If the intensity effects (petro and wood) are positive, the MRC is increased by growth in direct petroleum or wood-based carbon inputs to industry. If the supply chain effect is positive, the production structure through the supply chain emits more $\mathrm{CO}_{2}$ emissions or needs more petroleum/wooden materials intermediately. On the other hand, the pattern effect and volume effect are considered demand side indicators. If the pattern effect is positive, the relative consumption share of total consumption increases, indicating that related commodities are becoming more common among consumers, reflected by the maturing of the supply side (i.e., new production methods and reducing production costs) and/or more elasticity due to household income growth. If the volume effect is positive, the total consumption volume per capita increases. Finally, the size effect, distribution effect, and household effect measure the contribution of demographic changes to the EC and MRC. If the size effect is positive, EC and MRC are increased by growth in the average family size. If the distribution effect is 

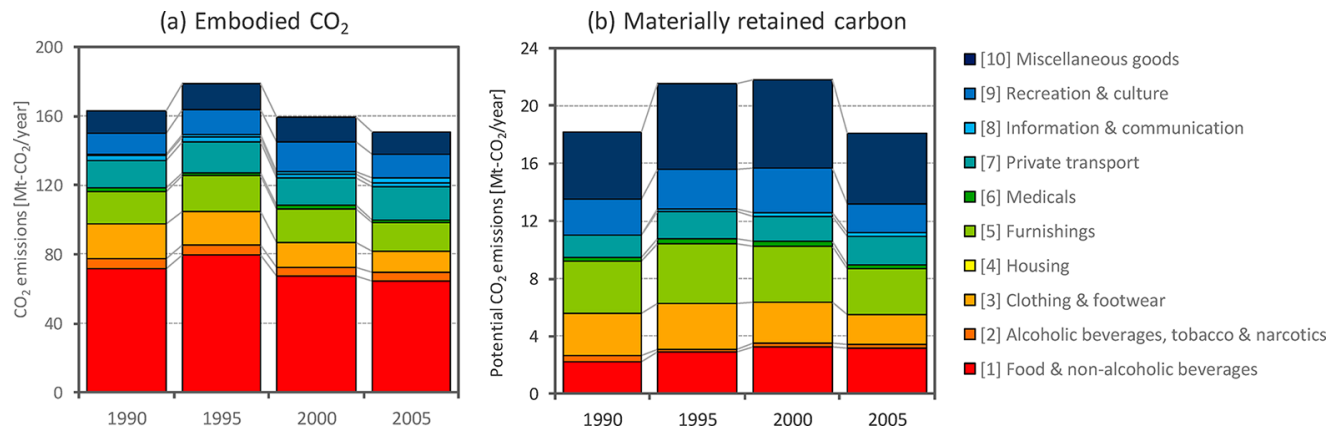

Figure 2. Structures of embodied $\mathrm{CO}_{2}$ (a) and materially retained carbon (b) induced by Japanese household consumption during 1990-2005.

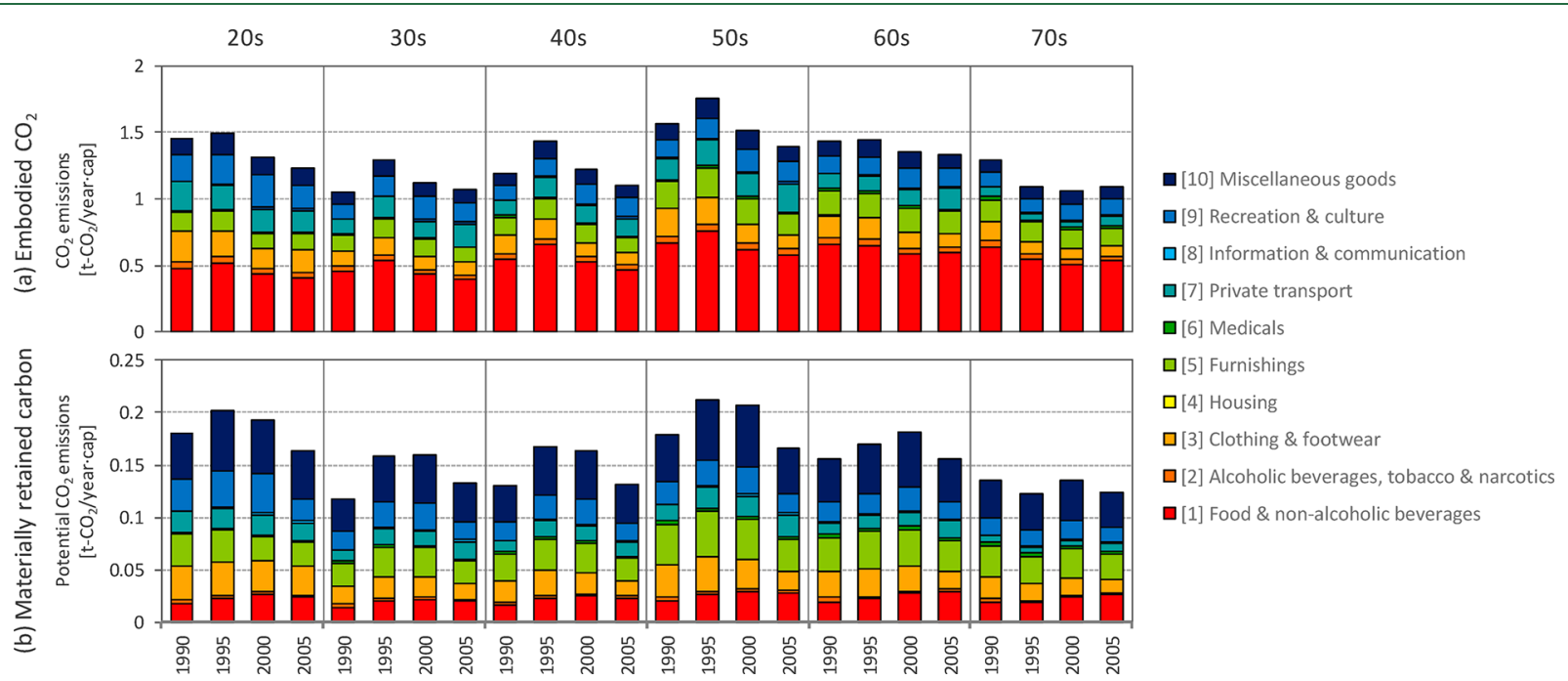

Figure 3. Per-capita embodied $\mathrm{CO}_{2}$ (a) and materially retained carbons (b) among age of the householders.

positive, EC and MRC are increased by changes in the distribution of householder ages (e.g., the proportion of elderly households to total household numbers). If the household effect is positive, EC and MRC are increased by growth in the number of households.

\section{RESULTS}

3.1. Time-Series Embodied $\mathrm{CO}_{2}$ Emissions and Materially Retained Carbon by Household Generation during 1990-2005. The EC and MRC for goods consumed by Japanese households during 1990-2005 were estimated as shown in Figure 2. To improve visibility, results are presented in line with 10 categories based on the UN COICOP (Classification of Individual Consumption by Purpose), aggregated from 397 commodities in the TJIO. These are [1] food and nonalcoholic beverages, [2] alcoholic beverages and tobacco, [3] clothing and footwear, [4] housing, [5] furnishings, [6] medical supplies, [7] private transport, [8] information and communication, [9] recreation and culture, and [10] miscellaneous goods. Hereafter, detailed commodities are described in italics (detailed information is in Table S3).

EC accounted for between 162 and $178 \mathrm{Mt}-\mathrm{CO}_{2}$, with the lowest amount of emissions during the target period observed in the year 2005. The major contributors to EC were food and nonalcoholic beverages, private transport, and furnishings. Note that electricity and fossil fuels such as gasoline, which contribute heavily toward EC were excluded due to the established need for a comparison with MRC. In addition, housing includes coal, kerosene, and heating gases (LPG and city gas); however, direct $\mathrm{CO}_{2}$ emissions is a result from their consumption, although the influence on total $\mathrm{CO}_{2}$ emissions is not considered. The EC peaked in 1995 and, after that, continued to decrease until 2005. These trends may be due to the postbubble economy in which Japan adopted government intervention policies to stimulate the recovery of the economy and increase household consumption, and the change in policy direction from economic stimulus to economic constraint (including raising the consumption tax and increasing medical expenses in 1997). ${ }^{56}$

On the other hand, the MRC for 1990 was estimated at 18.2 $\mathrm{Mt}-\mathrm{CO}_{2}$, growing to $21.8 \mathrm{Mt}-\mathrm{CO}_{2}$ by the year 2000. In 2005 however, it decreased by $3.7 \mathrm{Mt}-\mathrm{CO}_{2}$ to $18.1 \mathrm{Mt}-\mathrm{CO}_{2}$. For MRC, miscellaneous goods made the greatest contribution during the analyzed period and other major sources included furnishings, clothing and footwear, and food and nonalcoholic beverages. In particular, the growth of MRC for food and nonalcoholic beverages was significant. Investigating the major commodities in these three categories, plastic products, cosmetic products (in miscellaneous goods), household electric appliances (e.g., air-conditioners), wooden home equipment (in furnishings), soft drinks, vegetables (in food and nonalcoholic beverages) were found to be prominent. Passenger motor cars, knitted apparel, and automobile repairs also contributed markedly to MRC. The MRC for soft drinks and knitted apparel increased consistently from 1990, perhaps because plastic bottles and synthetic fibers made from petroleum chemical-based products such as polyethylene were increasingly popular and distributed 
(a) Embodied $\mathrm{CO}_{2}$

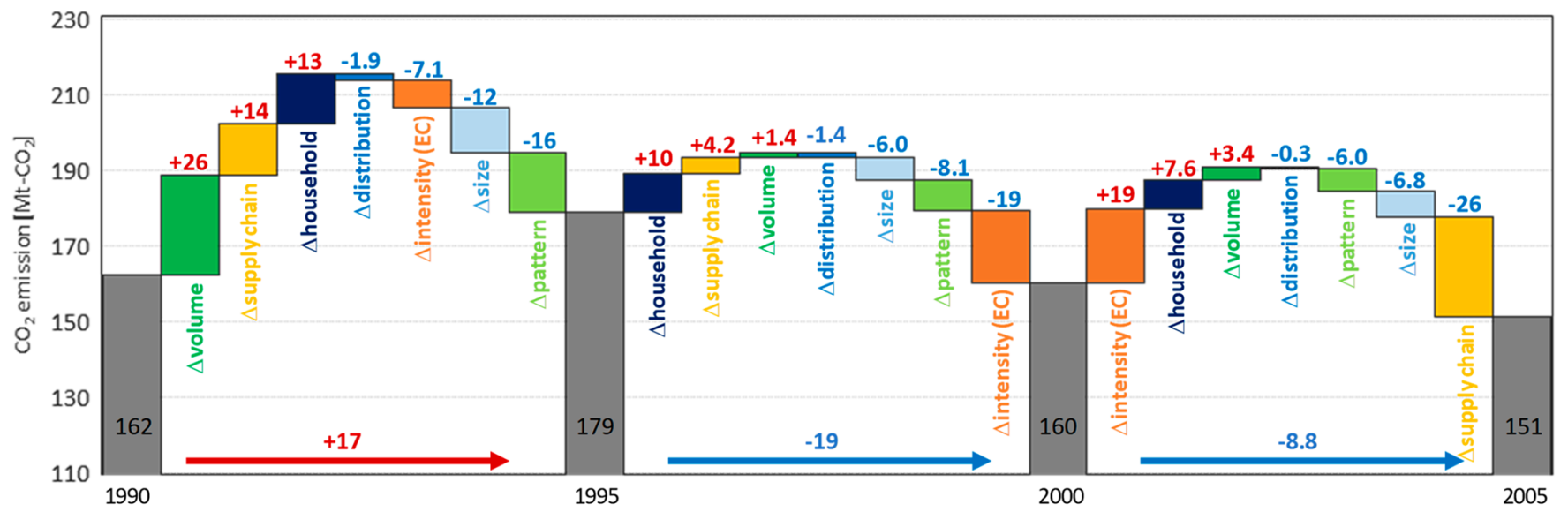

(b) Materially retained carbon

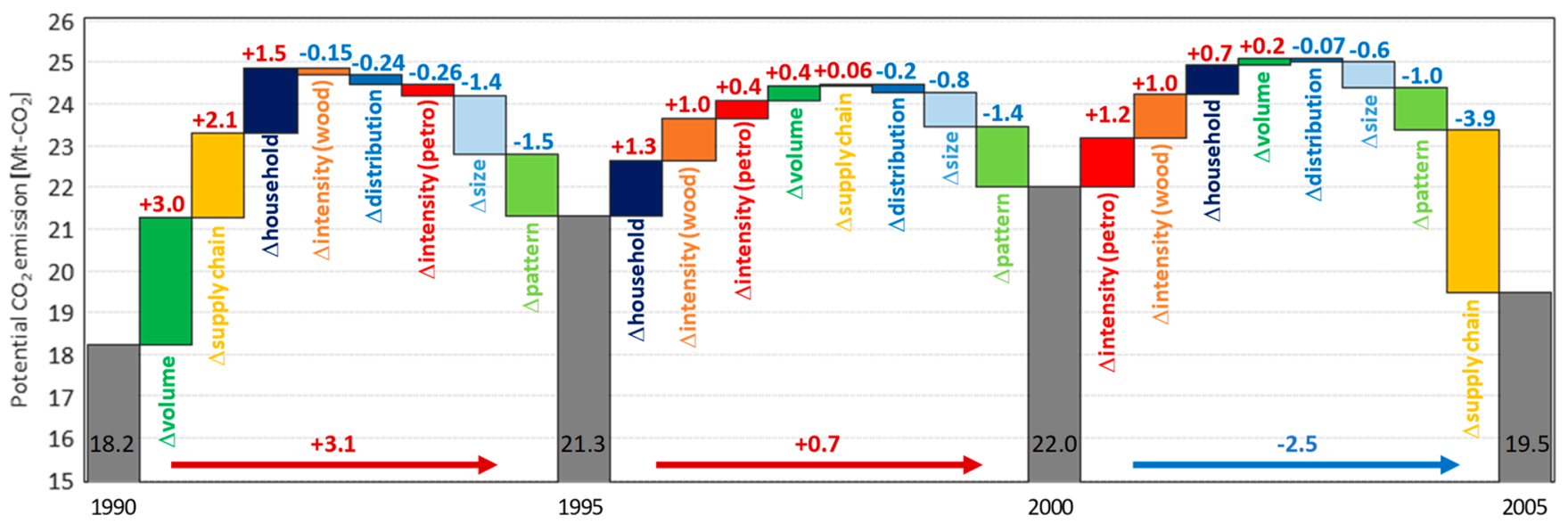

Figure 4. Decomposition results of the embodied $\mathrm{CO}_{2}$ (a) and materially retained carbon (b) during 1990-2005.

widely for convenient use. The above-mentioned commodities are considered key consumables for households which have the potential to prevent the release of carbon following initial consumption. Consumables in food and nonalcoholic beverages have a high potential for MRC due to high packaging rates.

3.2. Demographic Analysis for EC and MRC. Figure 3 represents the per-capita average EC and MRC by household bracket from 1990 to 2005 as estimated by dividing the total EC and MRC by household generation with the corresponding number of households $X$ average family size. From 1995, a decreasing trend in consumption expenditures per capita for goods was seen for households in their 20 s to 50 s, while those for the $60 \mathrm{~s}$ and $70 \mathrm{~s}$ were observed to increase since 1995 (Figure S3a in the Supporting Information). On the other hand, the total consumption expenditure including services showed a predominantly increasing trend across all households, demonstrating that Japanese society is shifting toward a service-based economy (Figure S3b). For per-capita EC, householders in their 50s made the largest contribution in the analyzed period, mainly due to higher consumption of food and nonalcoholic beverages, furnishing, and private transport. The second and third highest per-capita ECs included those in their 20s and 60s, and the former's EC per capita was induced by clothing and footwear, private transport, and recreation and culture while the latter's was induced by food and nonalcoholic beverages and furnishings, similar to those in their 50s.
Consumption for clothing and footwear, recreation and culture, and miscellaneous goods (i.e., cosmetics and plastic products) by those in their 20s drove per-capita MRC moderately. It is also notable that the 50 s and 20 s age groups played a key role in the contribution of information and communications to their MRC although this level is trivial among investigated categories. Looking at the elderly households (60s and 70s), per-capita MRC for alcohol beverages and tobacco and medical supplies were markedly high compared with other household brackets.

Compared to the per-capita EC per unit of consumption expenditure among household brackets, the per-capita MRC showed contrasting results as illustrated in Figure S4 (in the Supporting Information). Note that all MRC per unit of consumption expenditures by household generation in 2005 were higher than those in 1990, in contrast to EC per unit of consumption expenditure. The elderly households tended to have a larger per-capita EC per unit of consumption expenditure, implying that they were more likely to consume carbon intensive goods such as household electric appliances (5.8 $\mathrm{kg}-\mathrm{CO}_{2} /$ capita and $5.0 \mathrm{~kg}-\mathrm{CO}_{2} /$ capita, respectively) and medicaments (2.4 $\mathrm{kg}-\mathrm{CO}_{2} / \mathrm{capita}$ and $2.7 \mathrm{~kg}-\mathrm{CO}_{2} /$ capita, respectively) for their lifestyles. On the other hand, a larger per-capita MRC per unit of consumption expenditure was shown for younger households (20s and 30s). This implies that these groups, which consist mainly of single member households and family households with young children are 
(a) Embodied $\mathrm{CO}_{2}$
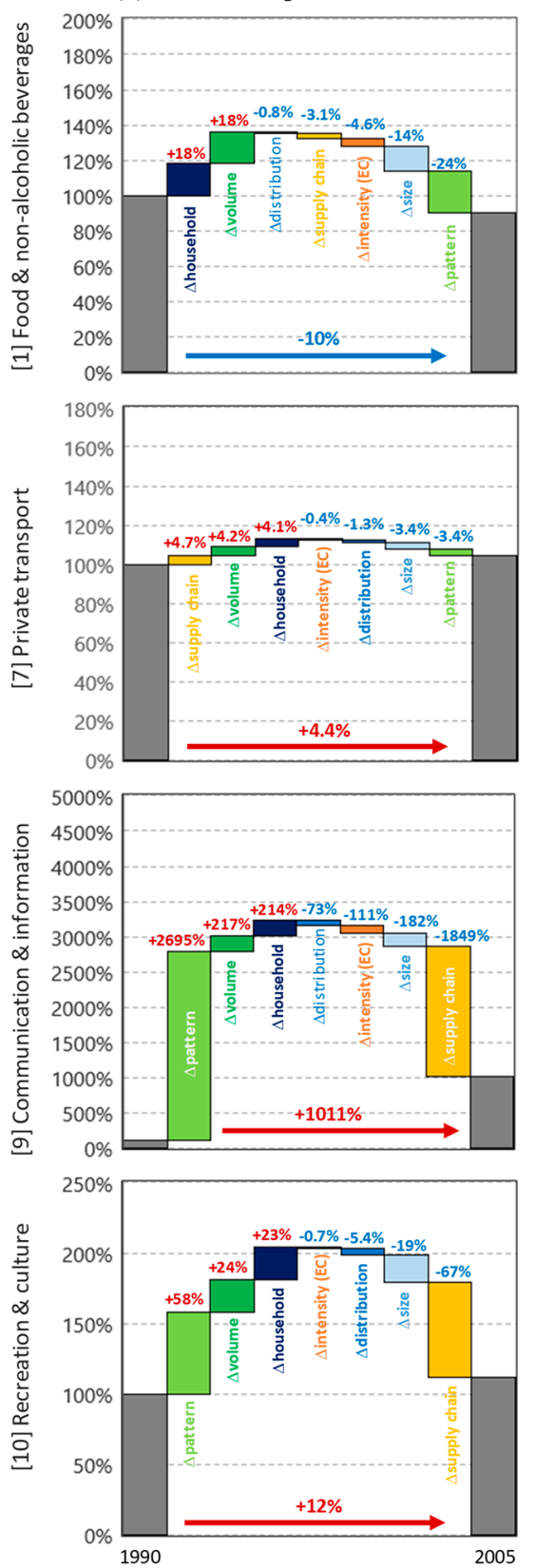

(b) Materially retained carbon
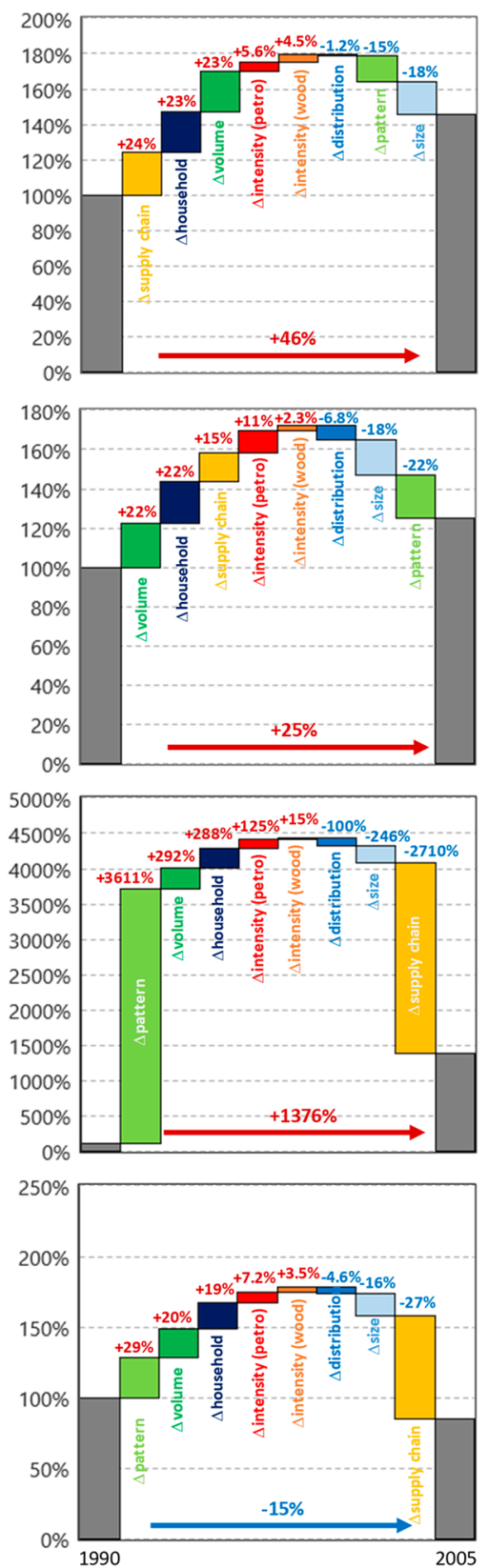

Figure 5. Decomposition results of the embodied $\mathrm{CO}_{2}$ (a) and materially retained carbon (b) for food, private automobiles, communication and information, and recreation and culture between 1990 and 2005.

more likely to consume goods with high levels of embedded carbon such as knitted apparel (14 kg- $\mathrm{CO}_{2} /$ capita and $8.5 \mathrm{~kg}$ $\mathrm{CO}_{2} /$ capita, respectively), soft drinks $\left(9.8 \mathrm{~kg}-\mathrm{CO}_{2} /\right.$ capita and $6.5 \mathrm{~kg}-\mathrm{CO}_{2} /$ capita, respectively), and miscellaneous leather products (5.9 $\mathrm{kg}-\mathrm{CO}_{2} /$ capita and $3.7 \mathrm{~kg}-\mathrm{CO}_{2} /$ capita, respectively).

3.3. Driving Forces of Change in Embodied $\mathrm{CO}_{2}$ Emissions and Materially Retained Carbon. The decomposition results of EC and MRC between 1990 and 2005 detailing the underpinning drivers are presented in Figure 4. Two drivers are considered influential, namely, the supply and demand sides within demographics. From the supply side, changes in direct $\mathrm{CO}_{2}$ emissions per output from industry (referred to as the intensity effect (EC)) initially decreased EC levels until 2000, after which they had a significant increasing effect. On the other hand, both direct petroleum and woodbased carbon inputs to industry (intensity effect (petro) and intensity effect (wood), respectively) drove an increase in MRC during 1995-2005. The intensity effect (wood) was a stronger positive driver than the intensity effect (petro) until 2000; however, the latter increased MRC substantially after 2000. This finding is supported by the wide ranging replacement of paper and steel products with alternative plastic-based materials and products. ${ }^{57,58}$ Note that in terms of achieving carbon-neutrality, reducing the intensity effect (petro) should be prioritized over the intensity effect (wood) because the disposal of wood products does not engender additional $\mathrm{CO}_{2}$ emissions. In contrast to the intensity effects, changes in the intermediate requirements for industries (supply chain effect) were initially a positive driver for both 
EC and MRC until 2000, subsequently becoming a negative driver in 2005, affecting the largest decrease thereafter. These trends are consistent with a previous study indicating that Japanese society shifted to a service economy during the investigated period. ${ }^{43}$

From the demand side, changes in consumption volume (i.e., the volume effect) was influential in increasing EC and MRC since 1990. Between 1990 and 1995, it was the largest positive driver among investigated drivers, affecting $\mathrm{EC}$ and MRC levels by $26 \mathrm{Mt}-\mathrm{CO}_{2}(+16 \%)$ and $3.0 \mathrm{Mt}-\mathrm{CO}_{2}(+17 \%)$, respectively. Although the effect decreased after 1995 compared to the 1990-1995 period, EC and MRC were increased overall. Changes in household consumption patterns (i.e., the pattern effect) negatively contributed to EC and MRC during all periods, implying that modernizing lifestyles negatively affect MRC levels. With regard to demographic trends, changes in the number of households (i.e., the household effect) consistently increased the amount of EC and MRC. The household effect impacts MRC levels by 1.5 (+8.4\%), $1.3(+4.7 \%)$, and $0.7 \mathrm{Mt}-\mathrm{CO}_{2}(+3.2 \%)$ for a total of $14 \%$ and EC levels by $13(+8.1 \%), 10(+5.6 \%)$, and $7.6 \mathrm{Mt}-$ $\mathrm{CO}_{2}(+4.8 \%)$ for a total of $19 \%$, during 1990-1995, 19952000, and 2000-2005, respectively. On the other hand, changes in household distribution (i.e., the distribution effect) and household size (i.e., the size effect) decreased EC and MRC consistently throughout the measured period. The influence of the distribution effect was very small on both compared to the population effect. However, both negative effects reduced in size over time. The combination of these demographic effects therefore was almost unnoticeable, implying that the demographic structure due to aging is not likely to substantially impact MRC and EC.

3.4. Key Sectors for Reducing Embodied $\mathbf{C O}_{2}$ Emissions and Materially Retained Carbon. This section highlights key industries, instrumental on EC and MRC, disaggregating them according to 10 established categories (detailed in section 3.1). As a result, EC and MRC for most categories showed a declining trend in 2005 compared with 1990 levels. The exceptions were EC for private transport, information and communication, and recreation and culture. The MRC for food and nonalcoholic beverages, private transport, information and communication, and miscellaneous goods also showed an increasing trend in 2005.

Figure 5 highlights a comparison of the drivers of EC and MRC for the four categories of food and nonalcoholic beverages, private transport, communication and information, and recreation and culture between 1990 and 2005. For food and nonalcoholic beverages, the household effect and volume effect engendered an increase in EC but the total amount decreased during the period, which is consistent with the previous study arguing the impact of aging demographics on food demands. ${ }^{19}$ This indicates that a reduction in upstream carbon emissions has been made from the point of view of demand side management. However, total MRC increased in contrast to EC, because of both the above-described effects and due to the supply chain and intensity effects which were both decreasing drivers of EC. The large contribution of supply chain effects rather than intensity effects is associated with a broad utilization of plastic bags and packaging. The methods used for packing, transporting, and delivering food to consumers through the supply chain needs to be reviewed (i.e., to avoid excessive packaging). In comparison, the EC and MRC of private transport increased in 2005 compared to 1990.
This is because of the increased utilization of plastic materials in automobiles due to the necessity for light-weighting to improve fuel efficiency. ${ }^{57}$ In addition, carbon-fiber reinforced plastic (CFRP) is one promising lightweight material for automobiles as a substitute for steel without sacrificing strength. ${ }^{59}$ The supply chain effect had an increasing impact, while the intensity effect had a mixed impact.

On the demand-side, the volume effect played a dominant role on increases in EC and MRC for all categories, having a greater impact than intensity effects between 1990 and 2005 . In contrast, the pattern effect decreased both MRC and EC for most categories except information and communication and recreation and culture. For these categories, although the related consumption volume was increased, diversification of consumption engendered a lower EC and MRC. The fact that the pattern effect increased EC and MRC for both information and communication and recreation and culture may be related to a maturing of the goods market, considering that cellular phones, portable music players, and personal computers are all products in these categories. For instance, the diffusion rate of cellular phones increased from $0.70 \%$ to $75.2 \%$ between 1990 and $2005 .{ }^{60}$ On the other hand, the average household income level did not increase. ${ }^{45}$ The pattern of consumption in these categories therefore leads to an increase in MRC due to the variety of goods required. Further, the household effect increased EC and MRC for all categories, while distribution and size effects reduced them. The combination of net demographic effects (the sum of household, distribution, and size effects) identified a contrast between the two categories in Figure 5, implying that an aging, shrinking population influences an increase in EC and MRC for food and nonalcoholic beverages and a decrease for private transport after 1990 .

\section{DISCUSSION}

4.1. Policy Implications for Managing MRC. This study demonstrated the different trends for both MRC and nonservice EC by household attribute and identified trends for key industries in line with changes in demand-side factors within household consumption (i.e., consumption volume and pattern) cognizant of future demographic shifts (i.e., family size, household composition, and number of households) as well as supply side factors (i.e., direct carbon input of unit output and intermediate requirements of carbon for unit output). Although it was shown that EC outweighed MRC, a consideration of MRC is also important because this metric allows for the highlighting of hot spot areas of downstream $\mathrm{CO}_{2}$ embedded in products that have already been consumed. To avoid the release of $\mathrm{CO}_{2}$ that comes from carbon embedded in products, managing "hot spot" demands can effectively reduce MRC. As shown in Figure 3, for instance, households in their 20s and 50s are more likely to generate per-capita MRC related to clothing and footwear, while 60s and 70s households tend to generate MRC related to food and nonalcoholic beverages more than other households except for those in their 50s. Focusing on trends in MRC by category, food and nonalcoholic beverages, private transport, information and communication, and miscellaneous goods (mainly cosmetic products) increased during the investigated period. In particular, industries associated with food and nonalcoholic beverages increased their dependency on petroleum-based chemicals, even though they reduced upstream carbon emissions. It is therefore essential for effective reduction of 


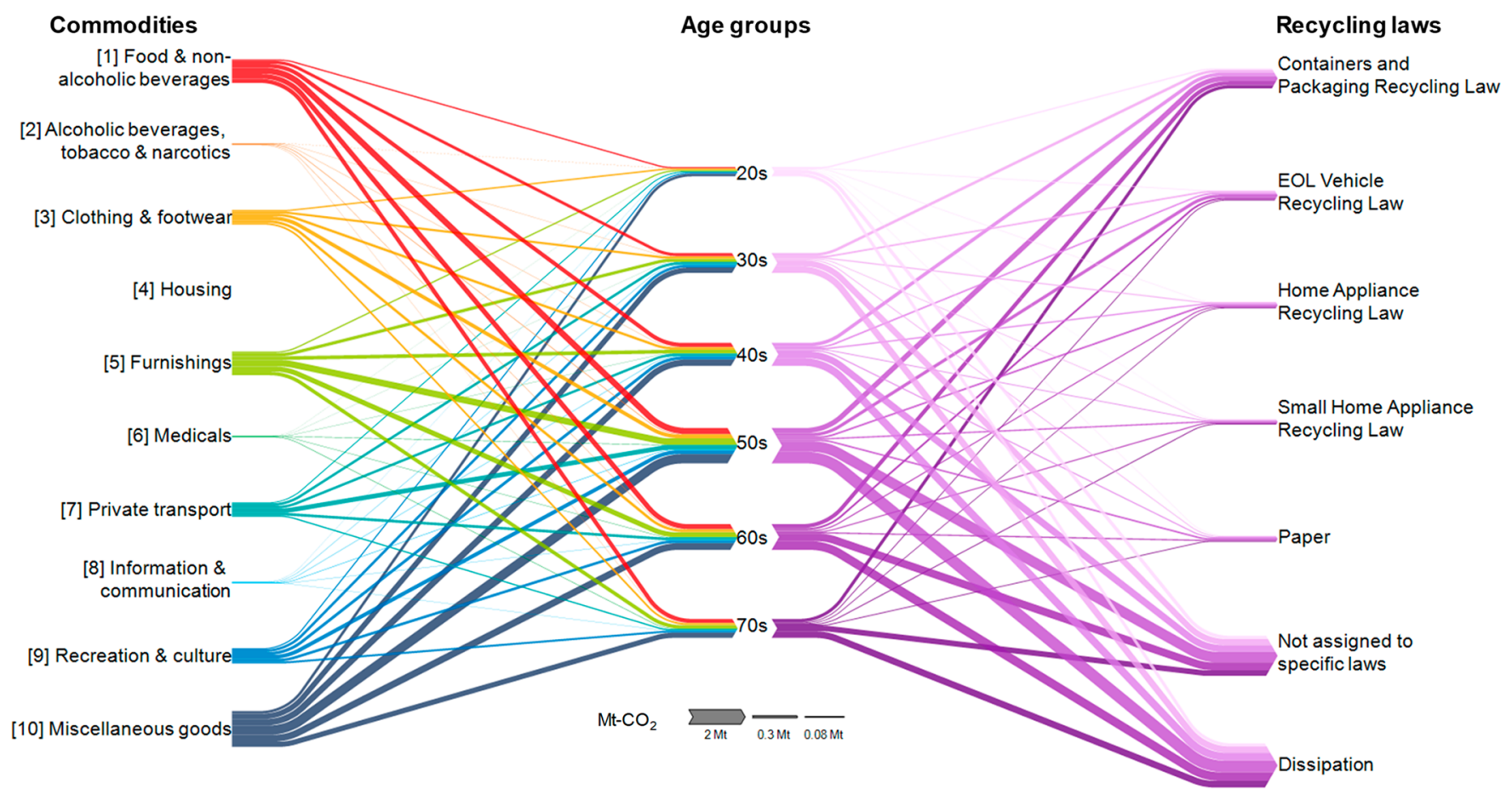

Figure 6. Carbon flows from goods to disposal via the six Japanese households in 2005. Descriptions on the right side represent the constitution of recycling potential by carbon-derived material with the Japanese municipal recycling laws.

MRC to increase efforts to reduce dependency on petroleumderived carbon by replacing petroleum-based chemicals with biomass derived products, paying attention to the trade-offs between land use and water resources. ${ }^{4,27}$ On the other hand, lightweight solutions, i.e., the use of high tensile strength steel and/or aluminum alloys may possibly contribute to a reduction in some industries' $\mathrm{CO}_{2}$ emissions through their production and/or relative supply chains on the supply side. If this approach is considered preferential, the reduction of production intensity, particularly for petroleum would not always result in maximal $\mathrm{CO}_{2}$ emission reductions. It is therefore critical to focus on demand-side aspects for managing MRC according to the results of this study. In order to improve MRC reduction outcomes, a consideration of lifestyles to reduce relative consumption based on the $3 \mathrm{R}$ (reduce, reuse, recycling) principle is important.

Another important aspect is the implementation of strategies for preventing additional carbon from being released into the atmosphere after consuming products. With regard to upstream burdens, prior to product consumption, it was identified that the combination of collaborative ownership of appliances and tools, a shift toward service usage, and secondhand buying or renting could reduce multiple household footprints. ${ }^{27}$ In the same vein, it is vital to extend product lifetimes by not only technological improvements on the supply side but also through "reduce and reuse" actions on the demand side. Behavioral changes, such as using reusable bottles in preference to single-use bottles along with the purchasing of second-hand goods may be effective. Further, legislation to ban disposable plastic products and campaigns for informing consumers about the linkage between behavior and environmental impacts ${ }^{61}$ are also appropriate. In terms of efficiency, establishing proper waste management instead of the current energy recovery ${ }^{56}$ (e.g., automobile shredder residue $^{62}$ ) processes may be of greater importance than just reducing the amount of carbon in goods accumulated through the supply chain, as this approach could play an additional role as a storage medium for $\mathrm{CO}_{2}$ similar to CCS, unless wastes are incinerated. ${ }^{5}$ Hence, encouraging consumers to clean and precisely segregate materials for targeted recycling is considered essential in facilitating an efficient recycling system on the demand side.

In line with the above findings, in Japan, four recycling laws have been implemented to promote recycling in the household. These include the Container and Packaging Recycling Law, the Home Appliance Recycling Law, the Small Home Appliance Recycling Law, and the Vehicle Recycling Law. ${ }^{63}$ Figure 6 illustrates how much carbon for each type of goods, by category, can be collected from each household under these recycling regimes since 2005 . Note that in the detailing of Figure 6, values of MRC such as dissipated carbon (e.g., in cosmetics) are excluded (33\% of total MRC) using the filtering approach explained in the Supporting Information. If the laws work ideally toward MRC recovery (although the Small Home Appliance Recycling Law actually started in 2013), the maximum potential carbon recoverable from goods is estimated to be $36 \%$ of total MRC (i.e., $6.6 \mathrm{Mt}-\mathrm{CO}_{2}$ ). On the other hand, as also seen in Figure 6, there is still significant potential for further recovery of carbon from products including apparel and plastic products except for packaging (i.e., 5.5 $\mathrm{Mt}-\mathrm{CO}_{2}$ ) although these products are not covered by the four specific recycling laws (attributable to the amounts specified "not assigned to specific laws" in Figure 6). Hence, it would be effective to inform major contributors, younger and elderly households, to collect these products as well as to promote a system for their recovery and material recycling. If this portion from the above four recycling laws (36\% of total MRC) was also covered, the final potential $\mathrm{CO}_{2}$ emissions reduction would account for $0.9 \%$ of all Japanese GHG emissions in $2005\left(=100 \times 12 / 1352 \mathrm{Mt}-\mathrm{CO}_{2}{ }^{64}\right)$. Although 
this amount seems relatively small, it translates to $21 \%$ of GHG emissions $\left(=100 \times 12 / 58 \mathrm{Mt}-\mathrm{CO}_{2}{ }^{65}\right)$ toward meeting emission reduction targets for the Japanese residential sector in 2030 under the Paris Agreement. ${ }^{41}$ Additionally, ongoing collection of MRC from households will increase this contribution. It is therefore necessary to create recovery pathways by assisting collection from households, particularly for the elderly, whose contribution will increase markedly as demographics shift toward an aging society.

The Group of Twenty (G20) summit held in June 2019 discussed the need for tackling marine plastic litter and microplastic issues on a global scale. In their discussion, promoting a comprehensive lifecycle approach to prevent and reduce plastic litter in line with sustainable consumption and production and sound waste management was highlighted. ${ }^{66}$ Since plastics share a large part of MRC in household goods (33\%), discussions pertaining to the sustainable management of MRC in this study can positively contribute to the mitigation of the problem. In particular, for nations whose demographic trends indicate longer lifetimes and low birthrates such as is the case in Japan, food and nonalcoholic beverages, clothing and footwear, furnishings, and miscellaneous goods such as cosmetics should be prioritized, as they show an increasing trend due to net demographic effects, expected to accelerate in the future. The government should aim to involve consumers in the closed loop system as detailed above to promote and enhance appropriate recycling approaches.

4.2. Limitations and Future Directions. The approach used in this study has some limitations and an element of uncertainty with regard to the data and methodology employed. The data, methodological, and uncertainty limitations are detailed in the Supporting Information.

The discussion on the availability of MRC-recovering technology and its potential benefit in terms of $\mathrm{CO}_{2}$ emission reduction will be one of the important future directions of this study as well as stock accounting for MRC including a prediction of waste generation. Future work may provide strategies for additional carbon reduction.

\section{ASSOCIATED CONTENT}

\section{S Supporting Information}

The Supporting Information is available free of charge on the ACS Publications website at DOI: 10.1021/acs.est.9b02603.

Method, supplementary results of MRC for petrochemicals and woody materials, and limitations for estimating EC and MRC associated with household consumption, flowchart of the procedure of IO data preparation (Figure S1), flowchart of the procedure of the material composition analysis (Figure S2), per-capita consumption expenditure for nonservice goods and services during 1990-2005 (Figure S3), EC per unit expenditure and MRC per unit expenditure during 1990-2005 (Figure S4), MRCs derived from petrochemicals and woody materials during 1990-2005 (Figure S5), MRCs derived from petrochemicals and woody materials per unit expenditure during 1990-2005 (Figure S6), formdetermining sectors for petro-carbon and woody-carbon (Table S1), list of variables utilized for structural decomposition analysis to decompose the EC and MRC (Table S2), list of commodities and categories considered for estimating the EC and MRC (Table S3), and list of commodities excluded for estimating the EC and MRC (Table S4) (PDF)

List of sectors and their category attribution, carbon contents per unit of materials and products, filtered input coefficient matrices for inputs of products to products by analyzed years, filtered input coefficient matrices for inputs of materials to products by analyzed years, filter matrices to filter out losses during production and packaging for intermediate products, filter matrices to filter out no-physical transactions, and result of the calculation (XLSX)

\section{AUTHOR INFORMATION}

\section{Corresponding Author}

*E-mail: y-shigetomi@nagasaki-u.ac.jp.

ORCID $\odot$

Yosuke Shigetomi: 0000-0003-1384-0992

Hajime Ohno: 0000-0002-8826-3854

Andrew Chapman: 0000-0002-2519-2382

Keisuke Nansai: 0000-0002-2449-1874

Yasuhiro Fukushima: 0000-0002-1525-7242

\section{Notes}

The authors declare no competing financial interest.

\section{ACKNOWLEDGMENTS}

We would like to thank the anonymous reviewers for their helpful and constructive comments on this paper. This research was supported in part by JSPS Grant-in-Aid for Young Researcher (Grants 18K18228 and 18K18231), JSPS Grant-in-Aid for Research Activity Start-Up (Grant 16H07072), and the Environment Research and Technology Development Fund (Grant 3-1801) of the Environmental Restoration and Conservation Agency of Japan.

\section{REFERENCES}

(1) Jambeck, J. R.; Geyer, R.; Wilcox, C.; Siegler, T. R.; Perryman, M.; Andrady, A.; Narayan, R.; Law, K. L. Plastic Waste Inputs from Land into the Ocean. Science 2015, 347 (6223), 768-771.

(2) Wright, S. L.; Kelly, F. J. Plastic and Human Health: A Micro Issue? Environ. Sci. Technol. 2017, 51 (12), 6634-6647.

(3) Kim, J. S.; Lee, H. J.; Kim, S. K.; Kim, H. J. Global Pattern of Microplastics (MPs) in Commercial Food-Grade Salts: Sea Salt as an Indicator of Seawater MP Pollution. Environ. Sci. Technol. 2018, 52 (21), 12819-12828.

(4) Zheng, J.; Suh, S. Strategies to Reduce the Global Carbon Footprint of Plastics. Nat. Clim. Change 2019, 9 (5), 374-378.

(5) Ohno, H.; Sato, H.; Fukushima, Y. Configuration of Materially Retained Carbon in Our Society: A WIO-MFA-Based Approach for Japan. Environ. Sci. Technol. 2018, 52 (7), 3899-3907.

(6) Singh, B.; Strømman, A. H.; Hertwich, E. G. Environmental Damage Assessment of Carbon Capture and Storage. J. Ind. Ecol. 2012, 16 (3), 407-419.

(7) Akashi, O.; Hijioka, Y.; Masui, T.; Hanaoka, T.; Kainuma, M. GHG Emission Scenarios in Asia and the World: The Key Technologies for Significant Reduction. Energy Econ. 2012, 34 (SUPPL. 3), S346-S358.

(8) Seto, K. C.; Davis, S. J.; Mitchell, R. B.; Stokes, E. C.; Unruh, G.; Ürge-Vorsatz, D. Carbon Lock-In: Types, Causes, and Policy Implications. Annu. Rev. Environ. Resour. 2016, 41 (1), 425-452.

(9) Chapman, A. J.; Pambudi, N. A. Strategic and User-Driven Transition Scenarios: Toward a Low Carbon Society, Encompassing the Issues of Sustainability and Societal Equity in Japan. J. Cleaner Prod. 2018, 172, 1014-1024. 
(10) Fujimori, S.; Matsuoka, Y. Development of Estimating Method of Global Carbon, Nitrogen, and Phosphorus Flows Caused by Human Activity. Ecol. Econ. 2007, 62 (3-4), 399-418.

(11) Lauk, C.; Haberl, H.; Erb, K. H.; Gingrich, S.; Krausmann, F. Global Socioeconomic Carbon Stocks in Long-Lived Products 19002008. Environ. Res. Lett. 2012, 7 (3), 034023.

(12) Chen, S.; Chen, B. Network Environ Perspective for Urban Metabolism and Carbon Emissions: A Case Study of Vienna, Austria. Environ. Sci. Technol. 2012, 46 (8), 4498-4506.

(13) Chen, S.; Chen, B. Changing Urban Carbon Metabolism over Time: Historical Trajectory and Future Pathway. Environ. Sci. Technol. 2017, 51 (13), 7560-7571.

(14) Aryapratama, R.; Pauliuk, S. Estimating In-Use Wood-Based Materials Carbon Stocks in Indonesia: Towards a Contribution to the National Climate Mitigation Effort. Resour. Conserv. Recycl. 2019, 149, 301-311.

(15) Nansai, K.; Kagawa, S.; Kondo, Y.; Suh, S.; Nakajima, K.; Inaba, R.; Oshita, Y.; Morimoto, T.; Kawashima, K.; Terakawa, T.; Tohno, S. Characterization of Economic Requirements for a "Carbon-Debt-Free Country. Environ. Sci. Technol. 2012, 46 (1), 155-163.

(16) Hertwich, E. G. The Life Cycle Environmental Impacts Of Consumption. Econ. Syst. Res. 2011, 23 (1), 27-47.

(17) Ivanova, D.; Stadler, K.; Steen-Olsen, K.; Wood, R.; Vita, G.; Tukker, A.; Hertwich, E. G. Environmental Impact Assessment of Household Consumption. J. Ind. Ecol. 2016, 20 (3), 526-536.

(18) Ivanova, D.; Vita, G.; Steen-Olsen, K.; Stadler, K.; Melo, P. C.; Wood, R.; Hertwich, E. G. Mapping the Carbon Footprint of EU Regions. Environ. Res. Lett. 2017, 12 (5), No. 054013.

(19) Vásquez, F.; Vita, G.; Müller, D. Food Security for an Aging and Heavier Population. Sustainability 2018, 10 (10), 3683.

(20) Chomik, R.; Piggott, J. Population Ageing and Social Security in Asia. Asian Econ. Policy Rev. 2015, 10 (2), 199-222.

(21) Ottelin, J.; Heinonen, J.; Junnila, S. Carbon and Material Footprints of a Welfare State: Why and How Governments Should Enhance Green Investments. Environ. Sci. Policy 2018, 86, 1-10.

(22) Shigetomi, Y.; Nansai, K.; Kagawa, S.; Tohno, S. Changes in the Carbon Footprint of Japanese Households in an Aging Society. Environ. Sci. Technol. 2014, 48 (11), 6069-6080.

(23) Kronenberg, T. The Impact of Demographic Change on Energy Use and Greenhouse Gas Emissions in Germany. Ecol. Econ. 2009, 68 (10), 2637-2645.

(24) Chapman, A.; Shigetomi, Y. Visualizing the Shape of Society: An Analysis of Public Bads and Burden Allocation Due to Household Consumption Using an Input-Output Approach. Sci. Total Environ. 2018, 639, 385-396.

(25) Shigetomi, Y.; Nansai, K.; Kagawa, S.; Tohno, S. Fertility-Rate Recovery and Double-Income Policies Require Solving the Carbon Gap under the Paris Agreement. Resour. Conserv. Recycl. 2018, 133, 385-394.

(26) Akenji, L. Annexes to the Technical Report 1.5-DEGREE LIFESTYLES: Targets and Options for Reducing Lifestyle Carbon Footprints Annexes to the Technical Report 1.5-Degree Lifestyles: Targets and Options for Reducing Lifestyle Carbon Footprints, 2019.

(27) Vita, G.; Lundström, J. R.; Hertwich, E. G.; Quist, J.; Ivanova, D.; Stadler, K.; Wood, R. The Environmental Impact of Green Consumption and Sufficiency Lifestyles Scenarios in Europe: Connecting Local Sustainability Visions to Global Consequences. Ecol. Econ. 2019, 164, 106322.

(28) Hoekstra, R.; van den Bergh, J. C. J. M. Comparing Structural Decomposition Analysis and Index. Energy Econ. 2003, 25 (1), 39-64.

(29) Su, B.; Ang, B. W. Structural Decomposition Analysis Applied to Energy and Emissions: Some Methodological Developments. Energy Econ. 2012, 34 (1), 177-188.

(30) Román-Collado, R.; Colinet, M. J. Is Energy Efficiency a Driver or an Inhibitor of Energy Consumption Changes in Spain? Two Decomposition Approaches. Energy Policy 2018, 115, 409-417.

(31) Guan, D.; Hubacek, K.; Weber, C. L.; Peters, G. P.; Reiner, D. M. The Drivers of Chinese $\mathrm{CO}_{2}$ Emissions from 1980 to 2030. Glob. Environ. Chang. 2008, 18 (4), 626-634.
(32) Feng, K.; Davis, S. J.; Sun, L.; Hubacek, K. Drivers of the US $\mathrm{CO}_{2}$ Emissions 1997-2013. Nat. Commun. 2015, 6, 7714.

(33) Arto, I.; Dietzenbacher, E. Drivers of the Growth in Global Greenhouse Gas Emissions. Environ. Sci. Technol. 2014, 48, 53885394.

(34) Zhao, C.; Chen, B. Driving Force Analysis of the Agricultural Water Footprint in China Based on the LMDI Method. Environ. Sci. Technol. 2014, 48 (21), 12723-12731.

(35) Fan, J.-L.; Wang, J.-D.; Zhang, X.; Kong, L.-S.; Song, Q.-Y. Exploring the Changes and Driving Forces of Water Footprints in China from 2002 to 2012: A Perspective of Final Demand. Sci. Total Environ. 2019, 650, 1101-1111.

(36) Wang, X.; Huang, K.; Yu, Y.; Hu, T.; Xu, Y. An Input-Output Structural Decomposition Analysis of Changes in Sectoral Water Footprint in China. Ecol. Indic. 2016, 69, 26-34.

(37) Weinzettel, J.; Kovanda, J. Structural Decomposition Analysis of Raw Material Consumption: The Case of the Czech Republic. J. Ind. Ecol. 2011, 15 (6), 893-907.

(38) Guan, D.; Su, X.; Zhang, Q.; Peters, G. P.; Liu, Z.; Lei, Y.; He, $\mathrm{K}$. The Socioeconomic Drivers of China's Primary $\mathrm{PM}_{2.5}$ Emissions. Environ. Res. Lett. 2014, 9 (2), 024010.

(39) Fujii, H.; Okamoto, S.; Kagawa, S.; Managi, S. Decomposition of Toxicity Emission Changes on the Demand and Supply Sides: Empirical Study of the US Industrial Sector. Environ. Res. Lett. 2017, 12 (12), 124008.

(40) Chapman, A.; Fujii, H.; Managi, S. Key Drivers for Cooperation toward Sustainable Development and the Management of $\mathrm{CO}_{2}$ Emissions: Comparative Analysis of Six Northeast Asian Countries. Sustainability 2018, 10 (1), 244.

(41) Shigetomi, Y.; Matsumoto, K.; Ogawa, Y.; Shiraki, H.; Yamamoto, Y.; Ochi, Y.; Ehara, T. Driving Forces Underlying SubNational Carbon Dioxide Emissions within the Household Sector and Implications for the Paris Agreement Targets in Japan. Appl. Energy 2018, 228, 2321-2332.

(42) Matsumoto, K.; Shigetomi, Y.; Shiraki, H.; Ochi, Y.; Ogawa, Y.; Ehara, T. Addressing Key Drivers of Regional $\mathrm{CO}_{2}$ Emissions of the Manufacturing Industry in Japan. Energy J. 2019, 40 (SI1), 233-258.

(43) Okamoto, S. Impacts of Growth of a Service Economy on $\mathrm{CO}_{2}$ Emissions: Japan's Case. J. Econ. Struct. 2013, 2 (1), 8 DOI: 10.1186/ 2193-2409-2-8.

(44) Ministry of Internal Affairs and Communications. Time-Series Japanese Input-Output Table, http://www.soumu.go.jp/toukei toukatsu/data/io/k gaiyou.htm.

(45) Ministry of Internal Affairs and Communications. NSFIE, National Survey of Family Income and Expenditure, https://www.e-stat. go.jp/stat-search $/$ database?page $=1$ \& toukei $=00200564 \&$ survey.

(46) Dalton, M.; O’Neill, B.; Prskawetz, A.; Jiang, L.; Pitkin, J. Population Aging and Future Carbon Emissions in the United States. Energy Econ. 2008, 30 (2), 642-675.

(47) O’Neill, B. C.; Dalton, M.; Fuchs, R.; Jiang, L.; Pachauri, S.; Zigova, K. Global Demographic Trends and Future Carbon Emissions. Proc. Natl. Acad. Sci. U. S. A. 2010, 107 (41), 1752117526.

(48) Chitnis, M.; Druckman, A.; Hunt, L. C.; Jackson, T.; Milne, S. Forecasting Scenarios for UK Household Expenditure and Associated GHG Emissions: Outlook to 2030. Ecol. Econ. 2012, 84, 129-141.

(49) Shigetomi, Y.; Nansai, K.; Kagawa, S.; Tohno, S. Influence of Income Difference on Carbon and Material Footprints for Critical Metals: The Case of Japanese Households. J. Econ. Struct. 2016, 5, 1 DOI: 10.1186/s40008-015-0033-4.

(50) Shigetomi, Y.; Nansai, K.; Kagawa, S.; Tohno, S. Trends in Japanese Households' Critical-Metals Material Footprints. Ecol. Econ. 2015, 119, 118-126.

(51) Huppes, G. Environmental Impacts of Consumption in the European Union. J. Ind. Ecol. 2006, 10 (3), 129-146.

(52) Miller, R. E.; Blair, P. D. Input-Output Analysis; Cambridge University Press: Cambridge, U.K., 2009, DOI: 10.1017/ CBO9780511626982. 
(53) Nakamura, S.; Nakajima, K.; Kondo, Y.; Nagasaka, T. The Waste Input-Output Approach to Materials Flow Analysis. J. Ind. Ecol. 2007, 11 (4), 50-63.

(54) Dietzenbacher, E.; Los, B. Structural Decomposition Techniques: Sense and Sensitivity. Econ. Syst. Res. 1998, 10 (4), 307-324.

(55) De Haan, M. A Structural Decomposition Analysis of Pollution in the Netherlands. Econ. Syst. Res. 2001, 13 (2), 181-196.

(56) Choi, Y.; Hirata, H.; Kim, S. H. Tax Reform in Japan: Is It Welfare-Enhancing? Japan World Econ. 2017, 42, 12-22.

(57) Miller, L.; Soulliere, K.; Sawyer-Beaulieu, S.; Tseng, S.; Tam, E. Challenges and Alternatives to Plastics Recycling in the Automotive Sector. Materials 2014, 7 (8), 5883-5902.

(58) Lange, J.; Wyser, Y. Recent Innovations in Barrier Technologies for Plastic Packaging-a Review. Packag. Technol. Sci. 2003, 16 (4), 149-158.

(59) Hottle, T.; Caffrey, C.; McDonald, J.; Dodder, R. Critical Factors Affecting Life Cycle Assessments of Material Choice for Vehicle Mass Reduction. Transp. Res. Part D Transp. Environ. 2017, 56, 241-257.

(60) International Telecommunication Union. World Telecommunication/ICT Development Report and database.

(61) Earth Day Network. Global Efforts to End Plastic Pollution: Single-Use Plastics, https://www.earthday.org/plasticban/ (accessed September 24, 2019).

(62) Sakai, S.; Yoshida, H.; Hiratsuka, J.; Vandecasteele, C.; Kohlmeyer, R.; Rotter, V. S.; Passarini, F.; Santini, A.; Peeler, M.; Li, J.; Oh, G.-J.; Chi, N. K.; Bastian, L.; Moore, S.; Kajiwara, N.; Takigami, H.; Itai, T.; Takahashi, S.; Tanabe, S.; Tomoda, K.; Hirakawa, T.; Hirai, Y.; Asari, M.; Yano, J. An International Comparative Study of End-of-Life Vehicle (ELV) Recycling Systems. J. Mater. Cycles Waste Manage. 2014, 16, 1-20.

(63) Ministry of the Environment. History and Current Situation of Waste Management in Japan, 2014.

(64) Greenhouse Gas Inventory Office of Japan, Center for Global Environmental Research Studies, National Institute for Environmental Studies. National Greenhouse Gas Inventory Report of Japan 2018, 2018.

(65) Ministry of the Environment. Plans for Mitigation in Climate Change, http://www.env.go.jp/earth/ondanka/keikaku/ onntaikeikaku-zentaiban.pdf.

(66) Ministry of the Environment. G20 Implementation Framework for Actions on Marine Plastic Litter, Japan, 2019. 